\title{
Ökologie- und marktgerechte Entwicklungsprinzipien
}

\section{Ökologische Aspekte spielen in der Entwicklung von Werkstoffen bisher allen- falls eine untergeordnete Rolle. Die Aufnahme von Umweltaspekten in den Entwicklungsvorgang ist aber machbar, besonders die Formulierung der Anforderungen an neue Stoffe bietet Raum für ihre Integration. Ein never, systematischer Entscheidungsprozess kann den Vorgang unterstützen.}

$\mathrm{D}$ Von Michael Achard er im Rahmen einer nachhaltigen Entwicklung erhobene Anspruch einer zunehmenden Ökologisierung der Marktwirtschaft erfordert auch ein grundlegendes Überdenken von Entscheidungsprozessen bei der Entwicklung von Werkstoffen. Bislang setzen sich jedoch die relevanten Entscheidungsträger aus Wissenschaft und Praxis vielfach nur partiell und nicht systematisch genug mit diesem Anliegen auseinander. Derzeit werden im Rahmen der Werkstoffentwicklung, die sämtliche Tätigkeiten des Erwerbs von Wissen durch Werkstoffforschung bis hin zur eigentlichen technischen Realisierung des Entwicklungsvorhabens umfasst, vor allem naturwissenschaftliche und/ oder technische Detailfragen der Umsetzungsphase untersucht.

Trotz wichtiger Fortschritte auf diesen Gebieten ist zu kritisieren, dass bis heute häufig lediglich physikalisch-technische Anforderungen sowie Kosten im Entwicklungsprozess beachtet werden. Ökologische und ökonomische Kriterien, vor allem auch diejenigen, die sich nicht in physikalischen oder monetären Größen abbilden lassen, werden bei der Werkstoffentwicklung vielfach ausgeklammert. Ebenso werden oft nicht alle Lebensphasen von Werkstoffen untersucht. Im betriebswirtschaftlichen Schrifttum werden hauptsächlich die unternehmerische Forschung und Entwicklung im Allgemeinen oder Produktentwicklungsprozesse analysiert (1). Viele Erkenntnisse, die in diesen Untersuchungen gewonnen werden, lassen sich jedoch nicht ohne weiteres auf die Belange der Werkstoffentwicklung übertragen, weshalb spezifische Werkstoffentwicklungsprozesse generiert werden müssen.

Angesichts der geschilderten Problemsituation werden wichtige ökologische und damit einhergehende ökonomische Erfolgspotenziale der Werk- stoffentwicklung nicht und/oder nicht umfassend genug erschlossen. Ausgehend von den genannten Defiziten werden deshalb im Folgenden einige elementare Ansatzpunkte aufgezeigt, wie Entscheidungsprozesse gestaltet werden können, damit der Prozess und der Output der Werkstoffentwicklung besser sowohl Anforderungen der natürlichen Systemumgebung gerecht werden als auch zur systematischen Erfiillung von betriebswirtschaftlichen Ansprïchen beitragen. Die Grundstruktur des hier vorgeschlagenen Werkstoffentwicklungsprozesses ist in Abbildung 1 illustriert.

\section{Qualitative ökologische Eigenschaften}

Im Prozess der Werkstoffentwicklung gilt es, auf Grundlage einer im Regelfall recht allgemein formulierten Entwicklungsaufgabe und einer daran anschließenden Anforderungsanalyse Soll-Eigenschaften zu extrahieren, die den nachgefragten Werkstoff möglichst genau beschreiben. Traditionell werden bei der Festlegung eines solchen SollEigenschaftsprofils vor allem physikalisch-technische und monetäre Parameter beachtet. Künftig sollte in ihrer Gesamtheit verstärkt die ökologische Verträglichkeit von Werkstoffen einbezogen werden. Die ökologische Verträglichkeit von Werkstoffen sagt aus, dass die Stoffflüsse von Werkstoffen über alle Lebensphasen in natürliche Kreisläufe integrierbar sind und die Stoffflüsse die Kreisläufe lediglich in vertretbaren Toleranzen beanspruchen. Ökologische Verträglichkeit beinhaltet somit Fragen nach der Ressourcenschonungsfähigkeit, der (Multi-)Funktionalität, dem Gefährdungspotenzial, der Recyclingfähigkeit und Konsistenzfähigkeit (3).

Nachdem die Soll-Eigenschaften des künftig einzusetzenden Werkstoffs bestimmt wurden, sind auf Grundlage des ermittelten Soll-Profils die Ist-
Eigenschaften im Leistungsprogramm befindlicher Werkstoffe mittels Verfahren der Werkstoffprüfung zu ermitteln. Die Kenntnis der Ist-Eigenschaften ist Voraussetzung zur Auswahl des Werkstoffs aus dem vorhandenen Werkstoffspektrum, der den Kundenwünschen am besten gerecht wird. Angesichts einer Vielzahl abzugleichender Informationen für eine Fülle an Werkstoffen erfolgt die Werkstoffauswahl in der Regel rechnergestützt. Ökologische Eigenschaften sind vom Wesen her häufig lediglich qualitativ oder verbalargumentativ bestimmbar. Zum Beispiel können Werkstoffe eine gute Stoffverträglichkeit, schlechte Konsistenzfähigkeit und geringe Ressourcenschonungsfähigkeit aufweisen. Solche Eigenschaften entziehen sich allerdings einer physikalisch-technischen Messbarkeit, weshalb sie in herkömmlichen Auswahlverfahren vielfach unbeachtet bleiben. Eine unscharfe Modellierung der Eigenschaften und Eigenschaftsbeziehungen durch die Anwendung von formalen Konzepten der Fuzzy-Set-Theorie, zum Beispiel Repräsentation in Zugehörigkeitsfunktionen und linguistischen Regeln, könnte künftig verstärkt deren Einbezug in Datenbanken oder Expertensystemen ermöglichen (4)

Abbildung 1: Prozess der Werkstoffentwicklung

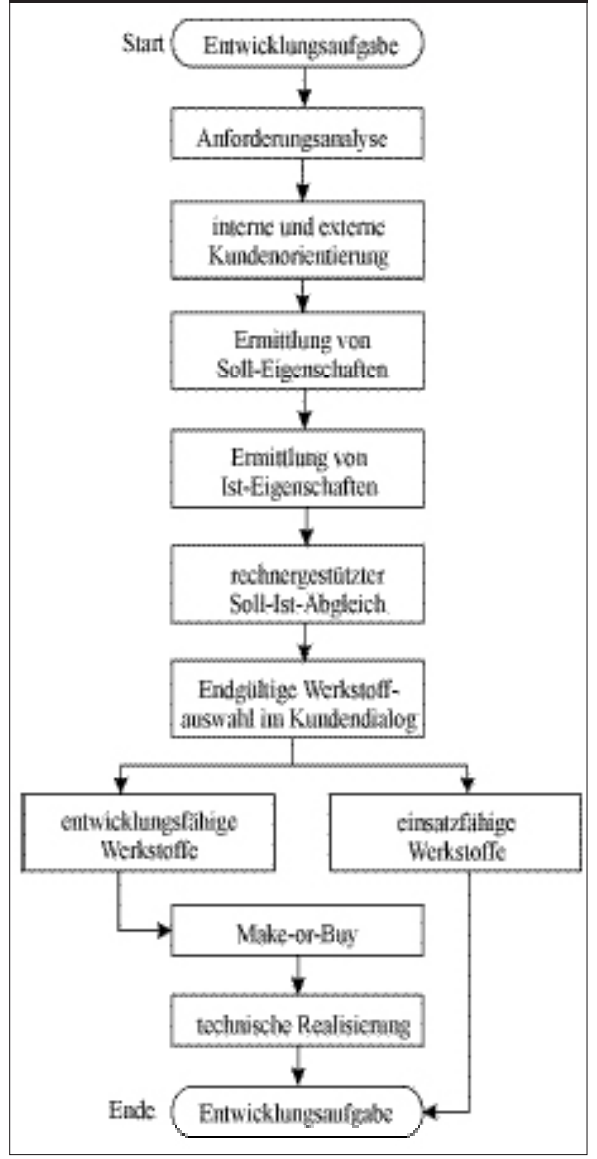




\section{- Interne und externe Kundenorientierung}

Die Kundenorientierung ist zweifelsohne ein Grunderfordernis des erfolgreichen unternehmerischen Auftritts am Markt. Auch in der aktuell vorherrschenden Philosophie der Maßwerkstoffentwicklung schlägt sich dies nieder, demnach bestimmte Eigenschaften von Werkstoffen zu einem kundengerechten Eigenschaftsprofil arrangiert werden. Der Kunde der Werkstoffentwicklung kann im Unternehmen oder unternehmensextern zu finden sein. (5)

In Abhängigkeit vom Standort des Kunden kann in den Differenzierungs- und Standardisierungsansatz unterschieden werden. Werkstoffdifferenzierung bedeutet, werkstoffliche Eigenschaften anhand spezieller unternehmensexterner Kundenbedürfnisse auszurichten. Werkstoffdifferenzierung prägt den heute vorherrschenden Trend der Entwicklung von Maßwerkstoffen, in deren Folge eine immer größer werdende Anzahl unterschiedlicher Werkstoffe am Markt angeboten werden. Die Werkstoffstandardisierung verkörpert das Pendant der Werkstoffdifferenzierung und zielt auf die weitestgehende Vereinheitlichung des Leistungsprogramms. Eine derart verstandene Standardisierung führt idealtypisch dazu, dass vom Unternehmen für den Markt im Extremfall nur noch ein einziger Werkstoff erstellt wird, weshalb regelmäßig lediglich durchschnittliche unternehmensexterne Kundenwünsche befriedigt werden können (6). Der bisher dominierende Fokus auf die Differenzierung vernachlässigt tendenziell Standardisierungsvorteile der Werkstoffentwicklung. So geht mit einer geringeren Werkstoffvielfalt im Regelfall eine höhere Recyclingfähigkeit von Werkstoffen einher. Ökonomische Vorteile gegenüber der Differenzierung bestehen zum Beispiel in den verbesserten Möglichkeiten bezüglich einer Verminderung der Nachfragemacht von Abnehmern, des Aufbaus von Markteintrittsbarrieren zum Schutz vor möglichen Konkurrenten bis hin zur Realisierung von Erfahrungskurveneffekten.

Entsprechend der hohen Bedeutung der Kundenorientierung ist in verschiedenen Phasen der Werkstoffentwicklung die Entscheidungsfindung durch Kundendialog zu unterstïtzen, insbesondere bei der Ermittlung von Soll-Eigenschaften und bei der endgültigen Werkstoffauswahl. Der wesentliche Vorteil einer engen Kundeneinbindung ist in der Vermeidung überdimensionierter Werkstoffe zu sehen. Überdimensionierungen liegen vor, wenn zusätzliche, nicht nachgefragte Werkstoffeigenschaften und/ oder übererfüllte Eigenschaftsniveaus angeboten werden. Die Vermeidung von Überdimensionierungen könnte sowohl einen schonenden Umgang mit ökologischen als auch mit ökonomischen Ressourcen zur Folge haben (7).

\section{Make-or-Buy}

Aus dem Abgleich der Soll- und Ist-Eigenschaften geht hervor, ob Werkstoffe einsatzfähig sind oder nicht. Wenn für die vorgesehene Anwendung einsatzfähige Werkstoffe ausgewählt werden, dann ist die jeweilige Entwicklungsaufgabe erfïllt und damit der gesamte Werkstoffentwicklungsprozess beendet. Auch wenn die begriffliche Auffassung weit verbreitet ist, dass aus Entwicklungsprozessen Innovationen hervorgehen, kann ein Ergebnis des Werkstoffentwicklungsprozesses durchaus auch ein bereits vorhandener, unter Umständen schon fast in Vergessenheit geratener Werkstoff sein. Im Falle der Existenz entwicklungsfähiger Werkstoffe sind vor der technischen Realisierung des Entwicklungsvorhabens noch weitere Entscheidungen im Werkstoffentwicklungsprozess zu treffen. Wegen der vielfältigsten Erscheinungsformen von Entwicklungsaufgaben kann zu Beginn eines Werkstoffentwicklungsprozesses nicht unbedingt eine sichere Aussage darüber getroffen werden, ob das unternehmenseigene Know-how der Aufgabe gewachsen ist. Vor allem entwicklungsbedürftige Werkstoffe, die erst in jüngster Vergangenheit und/ oder in Kooperation oder gar per Auftrag entwickelt wurden, können die unternehmensinternen Werkstoffentwicklungskapazitäten überbeanspruchen. Als betriebswirtschaftliche Grundsatzentscheidung ist deshalb zu klären, ob das weitere Vorgehen allein oder zusammen mit anderen Unternehmen gestaltet werden soll oder ob eine bestimmte Entwicklungsaufgabe nicht sogar gänzlich abzugeben ist. Vor allem die Kooperation des entwickelnden Unternehmens mit Unternehmen, die den Lebensweg von Werkstoffen begleiten wie Rohstofferzeuger, Anwender oder Recycling- und Beseitigungsunternehmen, sollte künftig an Priorität gewinnen. Dadurch könnten ökologische Informationen aus der Wertschöpfungskette bei der Werkstoffentwicklung besser explizit beachtet werden.

\section{Fazit und Ausblick}

Ausgehend von den eingangs skizzierten Defiziten wurden in den vorangegangenen Ausführungen Möglichkeiten dargelegt, den Prozess der Werk- stoffentwicklung sowohl nach ökologischen als auch grundlegenden ökonomischen Erfordernissen zu verbessern. Betont wurde vor allem, künftig zunehmend qualitative ökologische Informationen in die Entscheidungsfindung der Werkstoffentwicklung einzubeziehen sowie systematisch Grundsatzentscheidungen bezüglich der Kundenund Make-or-Buy-Orientierung in den Werkstoffentwicklungsprozess zu integrieren. Welche Entwicklungsalternative verfolgt werden soll, ist fallspezifisch durch deren ökologische und ökonomische Bewertung zu klären. Es ist davon auszugehen, dass in der Praxis sicherlich weniger die sture Befolgung eines einzelnen Entwicklungspfades, etwa Standardisierung, anzustreben ist. Vielmehr wird es sicherlich auf die sinnvolle Kombination beziehungsweise wechselseitige Abstimmung der skizzierten Entwicklungspfade ankommen.

\section{Anmerkungen}

(1) Brockhoff, K.: Forschung und Entwicklung. Planung und Kontrolle. München u.a. 1999 sowie Bennauer, U.: Ökologieorientierte Produktentwicklung. Eine strategisch-technologische Betrachtung der betriebswirtschaftlichen Rahmenbedingungen. Heidelberg 1994.

(2) Achard, M.: Kreislaufgerechte Werkstoffentwicklung Konzeption und Entscheidungsunterstützung. Dissertation, Hamburg 2004, Zabel, H.-U.: Ökologieverträglichkeit in betriebswirtschaftlicher Sicht. In: Zeitschrift für Betriebswirtschaft (ZfB), 4/ 1993, S. 351-372.

(4) Zadeh, L.A.: Fuzzy Sets. In: Information and Control, №. 8/ 1995, S. 338-353.

(5) Bruhn, M.: Implementierung einer kundenorientierten Unternehmensführung. In: Zeitschrift Führung + Organisation (Zf0), Jg. 72, 1/ 2003, S. 13-19.

(6) Mayer, R.: Strategien erfolgreicher Produktgestaltung : Individualisierung und Standardisierung. Wiesbaden 1993. (7) Fleig, J.: Umweltschutz in der schlanken Produktion. Analyse der schlanken Produktion in ihrer Relevanz für den betrieblichen Umweltschutz und Ableitung von Gestaltungsempfehlungen. Heidelberg 1998.

\section{Der Autor}

Dr. Michael Achard ist wissenschaftlicher Mitarbeiter am Demonstrationszentrum Kreislauffähigkeit von Werkstoffen am Institut für Polymerwerkstoffe (IPW). Kontakt: IPW, Geusaer Str. Geb. 131,

06217 Merseburg. Tel. 03461-462818,

E-Mail: michael.achard@dzkreis.uni-halle.de 
(c) 20I0 Authors; licensee IÖW and oekom verlag. This is an article distributed under the terms of the Creative Commons Attribution Non-Commercial No Derivates License (http://creativecommons.org/licenses/by-nc-nd/3.o/), which permits unrestricted use, distribution, and reproduction in any medium, provided the original work is properly cited. 